\title{
Stable Policies for Petri-Nets with Fluctuating Transition Processes
}

\author{
Costas Courcoubetis \\ Computer Science Department, University of Crete, \\ P.O.Box 1470, Heraklion, Crete, Greece 711-10 \\ and AT\&T Bell Laboratories, Murray Hill, NJ 07974, USA \\ Richard Weber \\ University Engineering Department, Management Studies Group, \\ Mill Lane, Cambridge CB2 1RX, UK
}

\begin{abstract}
Petri-Nets with finitely many transitions and places are considered. A transition process is associated with each transition that, clescribes the production and consumption of tokens when the transition is fired. Under certain assumptions about the fluctuation of the above processes and for various models of the underlying Petri-Net, we derive conditions for the existence of firing policies under which the number of tokens in the net satisfies some stability conditions.
\end{abstract}

\section{Petri-Nets with fluctuating transition processes}

Petri-Nets (PN's) have been widely used to model systems involving coordination among various components like multiprocessor systems and manufacturing facilities. In this paper we consider the usual specification of a PN, consisting of $m$ truthsitions and $n$ places, each with enough space to hold an arbitrary number of tokens. In this paper we speak of rumning the net in a certain mode. We suppose that running the net for one period in mode $k$ is equivalent to performing transition $k$ once, and changes the inventory level in place $i$ by $S_{k, i}$ tokens. It is a key idea in this paper that $S_{k, i}$ may be a random variable. For example, when modelling a manufacturing system this corresponds to there being unpredictable variations in the processes of demand and production. Let $x_{i}(t)$ denote the number of tokens in place $i$ at time $t$. Allowing $x_{i}(t)$ to assume negative values corresponds to allowing a backlog of tokens in place $i$. This models situations in which borrowing tokens of a certain type is possible provided there is a compensating production of tokens at a later time. $A$ negative value of $S_{k, i}$ indicates that transition $k$ consumes tokens from place $i$ and, in the case that $x_{i}(t)$ is negative, it increases the backlog of tokens in that place.

Imagine that at each discrete time $t,(t=0,1, \ldots)$, one mode of transition must be selected and the net run in that mode for the next time period. The central notion of the paper is that if the cost of carrying inventory of tokens is to be finite then the total number of tokens present should not remain large for long periorls of lime. We shall discuss two criteria that should be satisfied. 'The first criterion is the requirement that $P\left(\sum\left|x_{i}(t)\right| \rightarrow \infty\right)=0$. If this can be achieved by some operating policy. we say the system is weakly - stabilizable. If the inventory process converges to stationary regime then this implies that $a(t)$ has a well-clefined distribution. A stronger condition is to ask that the expected value of $\sum\left|x_{i}(t)\right|$ be bounded by a constant, uniformly in $t$. If this is possible we say that the system is stabilizable. Our aim is to give easily verifiable conditions under which the above model of a PN is weakly-stabilizable or stabilizable.

We begin with a description of the transition processes. Suppose that on the $t^{\prime}$ th occasion that the system is operated in mode $k$ the change in the inventory of tokens of type $i$ is $S_{k, i}(t)$. Let

$$
N_{k, i}(t)=\sum_{u=1}^{t} S_{k, i}(u)
$$

denote the cumulative change in the inventory of tokens of type $i$ due to the first $t$ periods of operation in mode $k$. Let $N_{k}(t)$ be the vector $\left(N_{k, 1}(t), \ldots, N_{k, n}(t)\right)$. Let $\mathcal{F}_{k}(s)$ be the $\sigma$-field generated by the entire history of the system up to the time mode $k$ is about

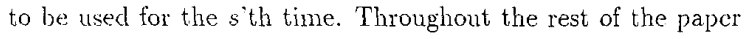
we shall make the following assumption regarding the processes $N_{k, i}(t)$.

Assumption A. For all $k, i, s$ and $t$

$$
E\left(\left|N_{k, i}(s+t)-N_{k, i}(s)-t \theta_{k, i}\right| \mid \mathcal{F}_{k}(s)\right) \leq g(t)
$$

where $g(t) / t \rightarrow 0$ as $t \rightarrow \infty$.

'This weak assumption simply states that the change to the inventory occasioned by $t$ periods of operation in mode $k$ will have an alsolute deviation from its mean whose expected value grows more slowly than linearly in $t$. One special case in which assumption A satisfied is when $S_{k, i}(t)$ is an ergodic Markov process for each $k, i$. Another is when $S_{k . i}(t)$ is a second order stationary process. Various kinds of renewal process also satisfy the assumption. As we will show in the next sections, the above condition is used for obtaining weak stabilizability. For obtaining stalilizability we will use the following condition on the second moments. Let

$$
U_{k, i}(t)=S_{k, i}(t)-E\left[S_{k, i}(t)\right]
$$

Assumption B. 'There exists a $C>0$ such that for all h: $, l_{1} s_{1}, s_{2}, l_{1}, l_{2}$,

$$
\because\left(\left\|\sum_{u_{1}=s_{1}}^{s_{1}+t_{1}} U_{k}\left(u_{1}\right)\right\|\left\|\sum_{u_{2}=s_{2}}^{s_{2}+t_{2}} U_{l}\left(u_{2}\right)\right\|\right) \leq C\left(t_{1}+t_{2}\right)
$$

Assumplion B holds if the variance of $S_{k . i}(t)$ is uniformly bounded and the covariance (or autocovariance when $k=l$ ) lxtwern $S_{k, j}(s)$ and $S_{l, j}(t)$ tends to zero sufficiently fast as ||$-s \mid \rightarrow \infty$. The assumption holds, for example, when the $\dot{S}_{k, i}(t)$ 's are independent, ir reducible, aperiodic Markov chajns. 
The present paper develops some new theory, which for the PN model allows one to make quite general assumptions about fluctuations of the transition processes. Under the conditions $\mathrm{A}$ and $\mathrm{B}$ we derive necessary and sufficient conditions for stability and give simple policies to achieve it. In previous work, described in section 5 , the policies that have been used to obtain stability of related systems have required the use of artificially complicated information about the state of the system and have been randomizing. The present paper presents simpler policies. In section 2 we present an algorithm for stabilizing our model when a backlog of tokens is allowed. In section 3 we consider the case in which backlogs are not allowed and the transitions of the net have a particular structure. In section 4 we prove that the conditions obtained for stabilizability are also necessary. Section 5 discusses related work and section 6 describes an algorithm for checking the sufficient conditions.

\section{Stability of a PN with backlogging}

In this section we consider a $\mathrm{PN}$ in which $x(t)$ can assume negative values, i.e. borrowing, or backlogging, of tokens is allowed.

Theorem 1 Consider the model described in section 1. Suppose that tokens can be backlogged, assumption $A$ holds, and the convex cone generated by $\theta_{1}, \ldots, \theta_{m}$ is the whole of $\mathcal{R}^{n}$. Then the system is weakly-stabilizable.

Proof. Suppose that at time $s$ the system has been operated in mode $k$ for $s_{k}$ periods, $s_{1}+\ldots+s_{m}=s$. The inventory level is $x(s)=\sum N_{k}\left(s_{k}\right)$. Consider the $L_{2}$ norm, $\|x(s)\|$. We shall prove the system is weakly-stabilizable by showing that there exists an increasing sequence of random times, $\left\{\sigma_{i}\right\}$, tending to infinity, such that both $E\left(\sigma_{i+1}-\sigma_{i}\right)$ and $E\left(\left\|x\left(\sigma_{i}\right)\right\|\right)$ are uniformly bounded for all $i$. Clearly, the second of these ensures that $P(\|x(t)\| \rightarrow \infty)=0$.

Let $H$ be the convex hull in $\mathcal{R}^{n}$ of the points $\theta_{1}, \ldots, \theta_{m}$. The origin must be in the interior of $H$, since as $\theta_{1}, \ldots, \theta_{m}$ are generators for $\mathcal{R}^{n}$ there must be points in $H$ in every direction from the origin. Choose an integer $t>0$ and probabilities $\alpha_{1}, \ldots, \alpha_{m}$, $\sum \alpha_{k}=1$, such that

$$
x(s)+t \sum_{k=1}^{m} \alpha_{k} \theta_{k}=0 .
$$

This is can be done since for large enough $t$ the vector $-x(s) / t$ lies in the interior of $H$. Now for each $k$ choose integers $t_{1}, \ldots, t_{m}$ such that $\left|t_{k}-t \alpha_{k}\right|<1$ and $t=t_{1}+\ldots+t_{m}$. Note that this implies,

$$
\begin{aligned}
\left\|x(s)+\sum_{k=1}^{m} t_{k} \theta_{k}\right\| & =\left\|x(s)+t \sum_{k=1}^{m} \alpha_{k} \theta_{k}+\sum_{k=1}^{m} t_{k} \theta_{k}-t \sum_{k=1}^{m} \alpha_{k} \theta_{k}\right\| \\
& \leq \sum_{k=1}^{m}\left\|\left(t_{k}-t \alpha_{k}\right) \theta_{k}\right\| \leq \delta_{2},
\end{aligned}
$$

where we use as a bound $\delta_{2}=\sum_{k, i}\left|0_{k, i}\right|$.

The next step is to bound $t$ in terms of $\|x(s)\|$. Note that $H$ lies within the ball of radius $\delta_{2}$. Consider a ball of radius $\delta_{1}>0$ that lies entirely within $H$. If $\|x(s)\|$ is sufficiently large then $\max \left\{\left|x_{i}(s)\right|\right\}$ will be large and we can choose the integer $t$ such that $\delta_{1}<\max \left\{\left|x_{i}(s)\right|\right\} / t<\delta_{2}$. Since $\|x(s)\| / \sqrt{n} \leq$ $\max \left\{\left|x_{i}(s)\right|\right\} \leq\|x(s)\|$, we have that for all $x(s)$ sufficiently large we can arrange to choose the $\alpha_{i}^{\prime}$ 's and $t$ to satisfy (1) and also so that

$$
\|x(s)\| /\left(\sqrt{n} \delta_{2}\right)<t<\|x(s)\| / \delta_{1} .
$$

Let $D=\{x:\|x(s)\| \leq L\}$, for $L$ chosen sufficiently large that we can ensure that (1) and (2) hold for integer $t$ where $x$ is not in $D$. Next we define the sequence of observation times $\left\{\sigma_{0}, \sigma_{1}, \ldots\right\}$, with $\sigma_{0}=0$. If $x\left(\sigma_{i}\right)$ is in $D$ run the system for one period in any mode and let $\sigma_{i+1}=\sigma_{i}+1$. If $x\left(\sigma_{i}\right)$ is not in $D$ run the system for $\tau_{i}=t$ periods with $t_{k}$ of these periods of transitions in mode $k$, where $t$ is chosen to satisfy (1) and (2) when $s=\sigma_{i}$ and the $t_{k}$ 's are as described above. In this case let $\sigma_{i+1}=\sigma_{i}+\tau_{i}$ and note the for $s=\sigma_{i}$ and $t=\tau_{i}$ we have that at time $s+t$, conditional on all the history up to time $s$,

$$
\begin{aligned}
& E(\|x(t+s)\|)=E\left(\left\|x(s)+\sum_{k=1}^{m}\left(N_{k}\left(s_{k}+t_{k}\right)-N_{k}\left(s_{k}\right)\right)\right\|\right) \\
& \leq\left\|x(s)+\sum_{k=1}^{m} \theta_{k} t_{k}\right\|+\sum_{k=1}^{m} E\left(\left\|N_{k}\left(s_{k}+t_{k}\right)-N_{k}\left(s_{k}\right)-\theta_{k} t_{k}\right\|\right) \\
& =\left\|x(s)+\sum_{k=1}^{m} \theta_{k} t_{k}\right\|+\zeta(t) \leq \delta_{2}+\zeta(t),
\end{aligned}
$$

where since each $t_{k}$ is less than $t$ it follows that $\zeta(t)$ is $o(t)$.

It follows from the left hand inequality in (2) that when $\|x(s)\|$ is sufficiently large $t$ will be large enough to imply $\zeta(t) / t \leq \delta_{1} / 2$; suppose $L$ is large enough to ensure this. Using the right hand inequality in (2) we have $\|x(s)\| /\left(t \delta_{1}\right) \geq 1$ and thus

$$
\begin{gathered}
E(\|x(t+s)\|) \leq \delta_{2}+\zeta(t)\|x(s)\| /\left(t \delta_{1}\right) \\
\leq \delta_{2}+(1 / 2)\|x(s)\| \leq p\|x(s)\|
\end{gathered}
$$

for some $\rho<1$. The final inequality holds for all $\|x(s)\|$ sufficiently large; again, assume $L$ has been chosen large enough.

From the above discussion we have that if $x\left(\sigma_{i}\right)$ is not in $D$ then $E\left(\left\|x\left(\sigma_{i+1}\right)\right\|\right)<\rho\left\|x\left(\sigma_{i}\right)\right\|$. On the other hand if $x\left(\sigma_{i}\right)$ is in $D$ then $E\left(\left\|x\left(\sigma_{i+1}\right)\right\|\right)$ is uniformly bounded, by $L+m n B$. So for all observation times

$$
E\left(\left\|x\left(\sigma_{i+1}\right)\right\|\right) \leq \rho E\left(\left\|x\left(\sigma_{i}\right)\right\|\right)+L+m n B .
$$

Thus if $\left\|x\left(\sigma_{0}\right)\right\| \leq(L+m n B) /(1-\rho)=M$, then (3) implies $E\left(\left\|x\left(\sigma_{i}\right)\right\|\right) \leq M$ for all $i$. This is sufficient to ensure weakstability. Furthermore, since we have $\delta_{1} t \leq\left\|x\left(\sigma_{i}\right)\right\|$, it follows that the expected value of the time between observations is less than $M / \delta_{1}$.

By imposing some extra conditions we can deduce the stronger results that the system is stabilizable.

Theorem 2 Suppose that assumption $B$ and the conditions of theorem 1 hold. Assume also that the transition processes are such that by using the policy described in proof of theorem 1 the process $x(t)$ is stationary. Then the system is stabilizable.

Proof. The stationarity assumption implies that both $\lim _{t \rightarrow \infty} E(\|x(t)\|)$ and $\lim _{t \rightarrow \infty} t^{-1} E\left[\sum_{s=0}^{t-1}\|x(s)\|\right]$ exist and have the same value. Using the observations times, $\left\{\sigma_{i}\right\}$, constructed in the proof of theorem 1 , we have

$$
\begin{aligned}
t^{-1} E\left[\sum_{s=0}^{t-1}\|x(s)\|\right] & \leq t^{-1} E\left[\sum_{i: \sigma_{i}<t} \sum_{s=\sigma_{i}}^{\sigma_{i}+1}\|x(s)\|\right] \\
& \leq t^{-1} \sum_{i: \sigma_{i}<t} E\left[E\left(\sum_{s=\sigma_{i}}^{\sigma_{i}+1}\|x(s)\| \mid \sigma_{i}, x\left(\sigma_{i}\right)\right)\right] \\
& \leq t^{-1} \sum_{i=0}^{t-1} E\left[E\left(\sum_{s=\sigma_{i}}^{\sigma_{i+1}}\|x(s)\| \mid \sigma_{i}, x\left(\sigma_{i}\right)\right)\right] .
\end{aligned}
$$

Now conditional on knowing $\sigma_{i}$ and $x\left(\sigma_{i}\right)$, the value of $\sigma_{i+1}$ is determined by the construction described in the proof of theorem 
1. Note that assumption $A$ implies that there exists a $B<\infty$ such that $E\left(\left|N_{k, i}(s+t)-N_{k, i}(s)-t \theta_{k, i}\right|\right) \leq B t$ for all $k, i, s$ and $t$. So from time $\sigma_{i}$ to time $\sigma_{i+1}$ the value of $E(\|x(s)\|)$ can increase by at most $m n B\left(\sigma_{i+1}-\sigma_{i}\right)$ and the above is

$$
\begin{aligned}
& \leq 1^{-1} \sum_{i=0}^{1-1} E\left[\left(\sigma_{i+1}-\sigma_{i}\right)\left(\left\|r\left(\sigma_{i}\right)\right\|+m n B\left(\sigma_{i+1}-\sigma_{i}\right)\right)\right] \\
& \leq t^{-1} \sum_{i=0}^{1-1} E\left[\left\|n\left(\sigma_{i}\right)\right\|^{2}\right]\left(1 / \delta_{1}+m n B / \delta_{1}^{2}\right),
\end{aligned}
$$

where the final inequality follows from $\left(\sigma_{i+1}-\sigma_{i}\right) \leq\left\|x\left(\sigma_{i}\right)\right\| / \delta_{1}$. Hence the system will be stabilizable if $E\left\|x\left(\sigma_{i}\right)\right\|^{2}$ is uniformly bounded for all $i$. To see that this is the case, consicer

$$
E\left(\left\|x\left(\sigma_{i}\right)\right\|^{2}\right) \leq\left(E\left\|r\left(\sigma_{i}\right)\right\|\right)^{2}+E\left(\left\|x\left(\sigma_{i}\right)-E\left[x\left(\sigma_{i}\right)\right]\right\|^{2}\right) .
$$

The first term on the right hand side is uniformly bounded, by theorem 1. Let $s_{k}$ be the number of times for which production mode $k$ has been run by time $\sigma_{i-1}$, and $t_{k}$ be the number of times mode $k$ is rum between times $\sigma_{i-1}$ and $\sigma_{i}$. The second term on the right hand side of (4) is

$$
\begin{aligned}
& E\left[E\left(\left\|\sum_{k=1}^{m} \sum_{t=s_{k}}^{s_{k}+t_{k}} U_{k}(t)\right\|^{2} \mid x\left(\sigma_{i-1}\right)\right)\right] \\
& \leq E\left[E\left(\sum_{k=1}^{m} \sum_{l=1}^{m}\left\|\sum_{t=s_{k}}^{s_{k}+t_{k}} U_{k}(t)\right\|\left\|\sum_{t=s_{l}} U_{l}(t)\right\| \mid x\left(\sigma_{i-1}\right)\right)\right] \\
& \leq C E\left[\sum_{k=1}^{m} \sum_{l=1}^{m}\left(t_{k}+t_{l}\right)\right] \\
& \leq m^{2} C E\left(\sigma_{i}-\sigma_{i-1}\right) \leq m^{2} C E\left\|x\left(\sigma_{i-1}\right)\right\| / \delta_{1},
\end{aligned}
$$

which is uniformly bounded by theorem 1 .

\section{Stability without backlogging}

3.1 PN's with producing and consuming transitions. In this section we suppose that inventories of tokens may not be negative. To do this we start with a restricted PN in which we can separate the notions of adding and subtracting from inventories. The system is said to be in a producing mode when any of modes $r+1, \ldots, m$ are used. Such modes only to add to the inventories: that is, $S_{k, i}(l)$ is nonnegative for each of these modes. The system is said to be in a consuming mode when one of modes $1, \ldots, r$, is used. In such a mode $S_{k, i}(t)$ is nonpositive. If the requests arising from running the system in a consuming mode for one time period can be met from inventory then this is done and the system is next run in consuming mode without any further production. However, if recqussts arise for tokens that are not in the inventory then before allowing further requests the system is run in producing morles until all outstanding requests are satisficel. Only alter satislying all recpuests arising from one period's operation in consuning mock do we consider requests arising from the next poriod of operation in consuming node. As before, when running the system we have a choice as to which of the modes we use. Stability is anchicver if it is possible to maintain inventories of bounderl itverage size.

Prior to stating theorem 3 we olseserve the following corollary of assumption A: there exists at constant ' $2 T$ ', that is inkpondent of the times and the history up to, and which for all ki, $i$, ()$_{k, i}>0$, it is the case that after ruming mode $k$ lor s periods the expected number of periods for which it is recfuired to rum mode $k$ until one more product $i$ is produced is less than $2 T$. We see this since if $t \theta_{k, i}>1$.

$$
\begin{aligned}
& P\left(N_{k, i}(s+t)-N_{k, i}(s)<1 \mid \mathcal{F}_{k}(s)\right) \\
& \quad=P\left(N_{k, i}(s+t)-N_{k, i}(s)-t \theta_{k, i}<1-t \theta_{k, i} \mid \mathcal{F}_{k}(s)\right) \\
& \quad=P\left(\left(N_{k, i}(s+t)-N_{k, i}(s)-t \theta_{k, i}\right)^{-} \geq t \theta_{k, i}-1 \mid \mathcal{F}_{k}(s)\right) \\
& \quad \leq E\left(\left(N_{k, i}(s+t)-N_{k, i}(s)-t \theta_{k, i}\right)^{-} \mid \mathcal{F}_{k}(s)\right) /\left(t \theta_{k, i}-1\right)
\end{aligned}
$$

By assumption $A$ the final term is less than $1 / 2$ for all $t$ greater than some integer $T$ sufficiently large. Thus for any history up to time $s$ the probability of producing at least one token of type $i$ during the next $T$ periods of use of mode $k$ is at least $1 / 2$. That is,

$$
P\left(N_{k, i}(s+t)-N_{k, i}(s) \geq 1 \mid \mathcal{F}_{k}(s)\right) \geq 1 / 2 .
$$

Since this holds for $s=u, u+T, u+2 T, \ldots$, we see that the expected time until one product of type $i$ is produced is less than $T=2 T$. Clearly, we can take $T$ large enough that this lolds uniformly for all $k$ and $i$.

Theorem 3 Suppose that for the model described above assumption $A$ holds for all $N_{k, i}(t)$ and the convex cone generated by $\theta_{1}, \ldots, \theta_{m}$ is the whole of $\mathcal{R}^{n}$. Then the system is weaklystabilizable. Furthermore, if the assumptions of theorem 2 hold the system is stabilizable.

Proof. The proof is a moclification of the proof for theorem 1. At every observation time $\sigma_{i}$ the inventory $x\left(\sigma_{i}\right)$ is nommegative. As in the proof of theorem 1 we solve (1) and show that for $\left\|x\left(\sigma_{i}\right)\right\|$ sufficiently large $t$ can be an integer with $\left\|x\left(\sigma_{i}\right)\right\| /\left(\delta_{2} \sqrt{n}\right)<t<\left\|x\left(\sigma_{i}\right)\right\| / \delta_{1}$. As in the proof of theorem 1 we choose $t_{k}$ such that $\left|\alpha_{k} t-t_{k}\right|<1$ and $t=t_{1}+\ldots+t_{m}$. If $x\left(\sigma_{i}\right)$ is in $D$ we run any producing mode for one period and set $\sigma_{i+1}=\sigma_{i}+1$. If $x\left(\sigma_{i}\right)$ is not in $D$ we first run each producing mode $k$ for $t_{k}$ periods, $k=r+1, \ldots, m$ and then run each consuming mode for $t_{k}$ periods, $k=1, \ldots, r$. Ignore for the moment the fact that in doing this some components of $x(t)$ may go negative. In a similar manner as in the proof of theorem 1 , we have that the inventory satisfies, for $s=\sigma_{i}$ and $t=\tau_{i}$,

$$
\begin{aligned}
E(\|r(t+s)\|) & =E\left(\left\|x(s)+\sum_{k=1}^{m}\left(N_{k}\left(s_{k}+t_{k}\right)-N_{k}\left(s_{k}\right)\right)\right\|\right) \\
& \leq \delta_{2}+\zeta(t) .
\end{aligned}
$$

However. invertories are not allowed to go negative. So suppose the effect of these $t$ periods is to cliange inventory of type $k$ tokens by $C_{k}$. The policy we shall use is augmented by the proviso that if following any period during which the system is run in consuming mode a token $j$ is requested that is not in the inventory, then a producing mode $k$ is chosen for which $\theta_{k, j}>0$ and the system is run in mocle $k$ until a token of type $j$ is produced. As demonstrated prior to the statement of the theorem, assumption A implies that the expected number of periods of production required to produce one more token of type $j$ is bounded for all $j$ by some $T<\infty$. The number of times we shall have to initiate extra production periods because of shortfalls in numbers of tokens of type $j$ is at most $\left(x_{j}\left(\sigma_{i}\right)+C_{j}\right)^{-}$. The result of all such additional production periods, say $\nu_{i}$ periods in total, due to shortages for all types of product, will be to produce some further number of tokens of type $j$, say $Z_{j}$. The next observation time is taken as $\sigma_{i+2}=\sigma_{i}+\tau_{i}+\nu_{i}$. It follows from assumption $A$ and (5) that 


$$
\begin{aligned}
E\left(\nu_{i}\right) & \leq T \sum_{j=1}^{n} E\left(\left(x_{j} \sigma_{i}\right)+C_{j}\right)^{-} \\
& \leq T E\left(\left\|x\left(\tau_{i}\right)+\sum_{k=1}^{m}\left(N_{k}(s+t)-N_{k}(s)\right)\right\|\right)
\end{aligned}
$$

and therefore $E\left(\nu_{i}\right)$ is $o(t)$. Thus $E\left(Z_{j}\right)$ is also $o(t)$. Let $C$ and $Z$ be the vectors with components $C_{j}$ and $Z_{j}$. The inventory at the next observation time will be

$$
\begin{aligned}
x\left(\sigma_{i+1}\right) & =\left(x\left(\sigma_{i}\right)+C\right)^{+}+Z-\left(x\left(\sigma_{i}\right)+C\right)^{-} \\
& \leq\left(x\left(\sigma_{i}\right)+C\right)^{+}+Z .
\end{aligned}
$$

Thus $\left\|x\left(\sigma_{i+1}\right)\right\|$ has an $o(t)$ bound of $\delta_{2}+\zeta(t)+\|E(Z)\|$. The theorem is completed along the same lines as theorem 1 . Under the assumptions of theorem 2, a similar proof shows that the system is stabilizable.

3.2 Deterministic transitions and fluctuating inputs. In this section we present a PN as a general model for a manufacturing system and apply some of the ideas from previous sections. We think of a manufacturing system that may be modeled by $n$ distinct quenes. Some queues are queues of orders for finished products. Other queues represent inventories of raw materials, parts and subassemblies, or work-in-progress waiting for processing at a given marhine. Items arrive from outside the system to these queues according to a stochastic process. The arriving items are supplies of raw materials and orders for finished products. As above, we let $x_{i}(t)$ be the number of items in queue $i$ at time $t$. We shall suppose that a mode of manufacture makes deterministic changes to the queues, subtracting items from some queues and adding items to others. Subtractions correspond to the consumption of raw materials or the use of intermediate subassemblies to build other subassemblies and finished products. A final assembly operation subtracts from certain queues the resources required to build a finished product and then also subtracts one unit from the queue of unfilled orclers for that product. A limiting constraint in this model is that the queues are not allowed to assume negative values, corresponding to the case in which a manufacturing mode cannot be executed if the subassemblies or materials it needs are not already available.

As in the previous section suppose that the $t$ 'th period of arrivals adds to $x$ the vector $S_{0}(t)$, which for this section is assumed to be nonnegative in every component. The modes of manufacture make deterministic changes to the quenes by addition to $x(t)$ of the vectors $S_{1}, \ldots, S_{n}$; these are fixed vectors that may have both positive and negative components. The above model corresponds to a PN where modes of manufacturing correspond to arbitrary deterministic transitions, and queues correspond to places. There is a unique fuctuating transition corresponding to mode 0 . This transition does not consume any tokens, and when fired for the t'th time it adds a random number $S_{0, i}(t)$ of tokens to place $i, i=1, \ldots m$. Note that if there is a nonnegative linear combinations of $S_{1}, \ldots, S_{m}$ equal to 0 , it is possible, given a large enough initial inventory, to construct a policy that stabilizes the system but never uses mode 0 . Since this does not model a practical alternative we shall only consider policies that select mode 0 infinitely often. We desire to stabilize the system by ensuring that for all $i$ and $t$ we have $E\left(x_{i}(t)\right)<B$ for some $B$.

Without loss of generality suppose it is exactly the first $r$ components of $\theta_{0}$ that are nonzero. Let

$$
\Gamma=\left\{x \in \mathcal{R}^{n}: x \leq 0 \text { and } x_{i}=0 \text { for } i>r\right\}
$$

Here $\Gamma$ is the set of nonpositive vectors that are only nonzero in components corresponding to queues to which $S_{0}(t)$ can add items. We call these first $r$ queues the input queues and the remaining queues the intermediate queues. Let

$$
\Lambda=\left\{x \in \Gamma: x=\sum_{k} \alpha_{k} S_{k}, \alpha_{k} \geq 0\right\}
$$

be the set of vectors in $\Gamma$ that can be represented as $E\left[\sum_{k} n_{k} S_{k}\right]$ for nomnegative integer-valued random variables $n_{k}$. It contains all those raudom combinations of the manufacturing modes whose effect is to not increase the size of the input queues and to leave the sizes of intermediate queues unchanged. We provide a lemma and a theorem that characterize stability.

Lemma $1 \Lambda$ is a finitely generated cone whose generators may be taken to be nonpositive integer-valued vectors. Each of these generators can be written as a positive linear combination with integer coefficients of the vectors $S_{1}, \ldots, S_{m}$.

Proof. Since the vectors $S_{1}, \ldots, S_{m}$ are integer-valued they generate a cone that can be written as $\{x: A x \geq 0\}$ for some matrix $A$ with integer coefficients. Similarly, $\Gamma=\{x: B x \geq 0\}$ for some $B$ with coefficients that are all $-1,0$ or 1 . Thus $\Lambda=$ $\{x: A y \geq 0, B x \geq 0\}$. Since the generators of $\Lambda$ must be rational vectors and they lie in the cone generated by $S_{1}, \ldots, S_{m}$, each of them can be expressed as a nonnegative linear combination of the $S_{k}$ 's. There must be such linear combinations in which the multipliers of the $S_{k}$ 's can be taken to be nonnegative rationals. It follows, by rescaling the generators, that there is a choice of generators for which the multipliers of the $S_{k}$ 's are nomegative integers.

Let $\theta_{k}^{0}$ and $\Lambda^{1}$ be the restrictions of $\theta_{k}$ and $\Lambda$ to their first $r$ components, i.e. the nonzero components of $\theta_{0}$.

Theorem 4 Suppose the arrival process $N_{0}(t)$ satisfes assumption $A$ and $-\theta_{0}^{1}$ lics in the interior of the conver cone $\Lambda^{1}$. Then the systrm is wealily-stabilizable. Furthermore, there exists a weakly-stabilizing policy under which the intermediate queue sizes are uniformly bounded. If the assumptions of theorem 2 hold then weakly-stablitizable may be strengthened to stabilizable.

Proof. Using lemma 1, let $S_{1}^{\prime \prime}, \ldots, S_{d}^{\prime}$ be the generators of $\Lambda$ such that $S_{l}^{\prime}=\sum_{k} \mu_{l k} S_{k}, l=1, \ldots, d$, where the $\mu_{l k}$ 's are nonnegative integers. We can associate with each $S_{l}^{\prime}$ a new manufacturing mode that corresponds to running mode $k$ for $\mu_{l k}$ times, $k=1, \ldots, m$. Consider the new system where the arrival mode is the same as before and the production modes are the ones corresponding to the $S_{l}^{\prime}, l=1, \ldots, d$. If we project the state of the system to one in which we watch only the input queues then we can weakly-stabilize this system while keeping the input queues nonnegative. This follows from an application of theorem 3 when there is a single producing mode corresponding to $S_{0}$ and $d$ request modes corresponding to the $S_{l}^{\prime \prime}$ s. Let $K=\max _{l}\left\{\sum_{k} \mu_{l k}\right\} \max _{k \geq 1, i}\left\{\left|S_{k, i}\right|\right\}$. One can see that if before running any mode $S_{l}^{\prime \prime}$ the size $x_{i}$ of each intermediate queue is greater than $K$, then while running the $S_{k}$ 's comprising $S_{l}^{\prime}$ this state will remain within the range $x_{i} \pm K$, and at the end reassume the value $x_{i}$. This implies that if the intermediate queues sizes are all at least $K$ initially we can weakly-stabilize the projection of the system onto its input queues and thereby weaklystabilize the complete system as well while ensuring that the intermoliate quene sizes are uniformly bounded. If we assume the conditions of theorem 2 a similar proof shows the system is stabilizable. 


\section{Necessary conditions for stabilizability}

In the previous sections we have given several variations of a model for a $\mathrm{PN}$ and derived sufficient conditions for these models to be weakly-stabilizable or stabilizable. In theorems 1-3 it was required that the cone generated by positive linear combinations of $\theta_{1}, \ldots, \theta_{k}$ be the whole of $\mathcal{R}^{n}$. This condition is necessary in the sense that if the cone generated by $\theta_{1}, \ldots, \theta_{m}$ is not the whole of $\mathcal{R}^{n}$ then, there are processes $S_{k}(t)$ for which the system cannot be statbilized. In this section we shall show that the sufficient condition is necessary when the processes $S_{k, i}(t)$ are independent Bernoulli processes. We discuss the necessity for theorem 2; the necessity argument is similar for the other theorems. Recall that the system is run in one of $k$ possible modes during each time period. Suppose the $\left\{S_{k, i}(t)\right\}$ are mutually independent random variables and $\left\{S_{k, i}(t)\right\}_{t=1}^{\infty}$ are identically distributed. Assumption A will hold.

Without loss of generality assume that if any of the $m$ modes are not available then the process is no longer stabilizable. Assume also that the inventory level of each token is affected by at least one mode. Inder the first assumption it is easy to see that to stabilize the system every mode must almost surely be used infinitely often. Suppose that there is such a policy, but the cone generated by $\theta_{1}, \ldots, \theta_{m}$ is not the whole of $\mathcal{R}^{\prime \prime}$. Then by Farkas' lemma there exists a $q$, which may be chosen to have integer coefficients, such that $q^{T} \theta_{k} \geq 0$ for all $k$. Choose such a $q$ and consider the integer-valued process $y(t)=q^{T} x(t)$. Note that if the system is run in mode $k$ during period $t$ we have $E[y(t+1)-y(t) \mid x(t)]=q^{T} \theta_{k} \geq 0$ and thus $y(t)$ is a submartingale and by the martingale convergence theorem it tends to a limit on every sample path. Now this must almost surely be a finite limit, since $|y(t)| \leq\|q\|\|x(t)\|$, and $E[x(t)]$ is uniformly bounded for all $t$ since it is produced by a stabilizing policy. Since $y(t)$ is an integer, its limit on almost every sample path must be an integer and $y(t)$ must be equal to this limit for all $t$ sufficiently large. But since we have assumed each mode is almost surely used infinitely often this is impossible unless on almost every sample path the incremental clange in $y$ when mode $k$ is used for the $t$ 'th time satisfies $q^{T} S_{k}(t)=0$ for all $t$ sufficiently large. Now if $q$ has only one nonzero component, say $i$, this would imply that on almost every sample path no mode effects the inventory of token $i$ for $t$ sufficiently large, contradicting our second assumption. If $q$ is nonzero in more than one component, say in all components $i \in I$, then it is clearly impossible to have $q^{T} S_{k}(t)=0$ unless the independent random variables $S_{k, i}(t), i \in I$, are almost surely constants for $t$ sufficiently large. Therefore, except in the special case that some modes clange the inventories of some tokens by deterministic amounts, the system cannot be stabilizable.

The above argument demonstrates that the sufficient condition in theorem 2 is necessary if the system is to be stabilizable for any $S_{k}(t)$ 's. The exception is when some of the $S_{k}$ 's are deterministic and have a positive linear combination that is zero. The necessity argument for the other stability theorems is similar.

\section{Relation to previous work}

The results in this paper are essentially the translation of the results in Courcoubetis and Weber [6] from the context of flexible manufacturing to that of Petri-Nets. Related results in the literature are the following. The notion of stabilizability has been previously studied in the context of on-line bin-packing problems in [2], [3], [4], [5] and for off-line packing in [8]. One can think of $n$ types of item that arrive to a bin-packing system and can be packed into bins in various ways. For example, a bin might be fully packed by either one item of each of types 1,2 and 4 , or by five items of type 3 . The idea is to pack items in bins, as they arrive, and ensure that the time-average number of partially full bins remains bounded. Clearly, this is similar to the Petri-Net formulation if we think of spaces for items as arising from a production process and the demand for these spaces as being generated by randomly arriving items. In [3] we gave a necessary and sufficient condition for a bin-packing system to be stabilizable when items arrive according to independent Bernoulli processes. In [4] this was more fully developed for several special types of packing configurations. In [2] Courcoubetis and Rothblum considered models in which rewards are obtained when different packing configurations used. Rhee and Talagrand [8] considered a model in which sizes of items are uniformly distributed over a subinterval $[a, b]$ of $[0,1]$ and a bin can contain any number of items whose sizes add to no more than 1 . They characterized the choices of $[a, b]$ for which the wasted space in partially full bins can be held finite in time average by using an off-line packing algorithm. In [5] we have considered stabilizing systems when there are side constraints on the frequencies with which bins may be packed in different ways. A production model with batch arrivals has been considered in [1].

\section{Discussion}

We have seen that if the models in this paper are reasonable for a particular application and we can estimate the vectors $\theta_{k}$, then theorems 1-4 provide criteria by which to test whether the system can operated in a manner that keeps expected inventory levels uniformly bounded through time. The criteria of the theorems can be tested using simple algorithms. In theorems 1-3 it was required that the cone generated by nonnegative linear combinations of $\theta_{1}, \ldots, \theta_{m}$ be the whole of $\mathcal{R}^{n}$. This holds if and only if $\theta_{1}, \ldots, \theta_{m}$ span $\mathcal{R}^{n}$ and there exist $\gamma_{1}, \ldots, \gamma_{m}$ such that $\gamma_{k}>1$ for all $k$ and $\sum_{k} \gamma_{k} \theta_{k}=0$. This can be checked by a linear program in $m$ variables. Theorem 4 required that $-\theta_{0}^{1}$ be in the interior of $\Lambda^{1}$. An algorithm to check this can be based on the following ideas. Let $\Omega=\left\{x \in \mathcal{R}^{n}: x_{i}=0\right.$ for $\left.i>r\right\}$ and define $I$ as the set of those indices $i>0$ for which there exists $\left\{\alpha_{k} \geq 0, k \neq i\right\}$ such that $\theta_{i}+\sum_{k \neq i} \alpha_{k} \theta_{k} \in \Omega$. The condition of theorem 4 is equivalent to requiring that the vectors $\left\{\theta_{i}, i \in I\right\}$ span $\Omega$ and there exist $\left\{\gamma_{i}>0, i \in I\right\}$ such that $\sum_{i \in I} \gamma_{i} \theta_{i}=-\theta_{0}$.

There are at least two important questions outstanding. Firstly, one would like to identify practical policies that achieve stability: The policies described in the proofs of theorems 1-4 can be implemented by relatively simple calculations, but they are wasteful in use of inventory space and certainly not rules-ofthumb. or methods one would recommend in practice. Clearly, it is important to minimize average inventory levels, not just to ensure their expected values remain finite. One would really like to find policies that stabilize the system and require minimum average inventory levels to clo so. Secondly, the maximum inventory size is likely to be constrained. Interestingly, we can say something about this sort of constraint when assumption $A$ is replaced by

Assumption C. For all $k, i, s$ and $t$

$$
\left|N_{k, i}(s+t)-N_{k, i}(s)-t \theta_{k, i}\right| \leq g(t)
$$

where $g(t) / t \rightarrow 0$ as $t \rightarrow \infty$.

Under this assumption it is straightforward to modify the proofs of theorems $1-4$ to show stability in the sense that $\left|x_{i}(t)\right|$ is bounded by a constant uniformly in $t$. 
Another practical consideration may be constraints on the rates at which some of the transition modes are used. For example, we might pose the problem of stabilizing the system subject to a constraint that a particular mode $k$ be used at rate $\Lambda_{k}$. This problem has been considered in [5] and similar results can be obtained for the models in this paper.

\section{Acknowledgement}

We are grateful to Alan Weiss for help in formulating assumption $B$ and the proof of theorem 2.

\section{References}

[1] Courcoubetis, C., Konstantopoulos, P., Walrand, J. and Weber, R.R., 1990. Stabilizing an uncertain production system, Queueing Systems: Theory and Applications, to appear.

[2] Courcoubetis, C. and Rothblum, U.G., 1990. On optimal packing of randomly arriving objects, Math. Operat. Res., to appear.

[3] Courcoubetis, C. and Weber, R.R., 1986a. Necessary and sufficient conditions for stability of a bin-packing system, $J$. Appl. Prob. 23, 989-999.

[4] Courcoubetis, C. and Weber, R.R. 1986b. A bin-packing system for objects from a finite set: necessary and sufficient conditions for stability and some applications, Proc. of 25 th IEEE CDC, Athens, Greece, December 1986.

[5] Courcoubetis, C. and Weber, R.R., 1990. Stability of online bin packing with random arrivals and long-run-average constraints, Probability in the Engineering and Information Sciences, to appear.

[6] Courcoubetis, C. and Weber, R.R., 1990. Stability of flexible manfacturing systems, Operations Research, to appear.

[7] Eaves, B.C. and Rothblum, U.G., 1988. A discrete time average cost flexible manufacturing and operator scheduling model solved by deconvexification over time, Operations Research 36, 242-247.

[8] Rhee. W.T. and Talagrand, M., 1988. Some distributions that allow perfect packing, J. Assoc. Comput. Mach. 35, 564-578. 